\title{
EL CAPITAL INTELECTUAL Y LA \\ CONTABILIDAD DEL CONOCIMIENTO: \\ ESTUDIO Y APLICACIONES EN EL PERÚ
}

Dr. Ernesto Augusto Polar Falcón*

\begin{abstract}
RESUMEN
El presente artículo tiene como objetivo básico incentivar a los Contadores Públicos y a los estudiantes universitarios, futuros Contadores Públicos, al estudio de los temas de Capital Intelectual y Contabilidad del Conocimiento, que ya son canales de aplicación profesional en el mundo.

Se presenta una secuencia metodológica de lectura bibliográfica, y de los trabajos nacionales interamericanos, como fuentes de conocimiento que motivarán a seguir buscando nuevas aportaciones científicas y profesionales sobre estos interesantes temas.

La mejor contribución a la profesión contable que se hace en este artículo es la presentación del cuadro titulado "Modelo de Información Complementaria sobre Capital Intelectual".
\end{abstract}

\section{INTRODUCCIÓN}

A partir de 1999 los Contadores Públicos del Perú tuvimos la oportunidad de leer libros y trabajos interamericanos relacionados con los temas de la Economía del Conocimiento, la Administración del Capital Intelectual y la Contabilidad del Conocimiento.

Paralelamente, en setiembre de 1998 el Comité de Normas Internacionales (IASC son sus siglas en inglés), publicó la NIC 38 - ACTIVOS INTANGIBLES, que "prescribe la contabilidad y la revelación de activos intangibles que no son tratados específicamente en otras Normas Internacionales de Contabilidad". También en la misma fecha el Comité de Contabilidad Financiera y Gerencial de la Federación
Internacional de Contadores publicó el estudio titulado MEDICIÓN Y ADMINISTRACIÓN DEL CAPITAL INTELECTUAL: UNA INTRODUCCIÓN; este trabajo fue más conocido como el ESTUDIO 7.

L. Thurow" nos dice que: "el conocimiento es la nueva base de la riqueza. Nunca lo había sido. En el pasado, cuando los capitalistas hablaban de su riqueza se referían a las fábricas, a los equipamientos y a los recursos naturales que poseían. En el futuro, cuando los capitalistas hablen de su riqueza estarán refiriéndose al control que poseen sobre el conocimiento". Mejor se expresarían si se habla del "control del conocimiento".

Por lo expuesto, el presente artículo hace el esfuerzo profesional de mostrar uno de

* Profesor Principal y Director del Instituto de Investigaciones de Ciencias Financieras y Contables

1 Lester Thurow. Construir Riqueza. Editorial Bergara-El Capital Intelectual-Sub. Tema 1.1.1. Capital Intelectual-XXIV Conferencia Interamericana de Contabilidad. 
los caminos que pudiera seguirse para ingresar al estudio de estos temas de vigente actualidad, así como enterarse de los avances logrados con la lectura de los últimos trabajos interamericanos y los artículos publicados en revistas especializadas de investigación contable que hemos tenido la suerte de recibir.

Invocamos al lector a trazarse también un camino de estudio por cuanto se puede pronosticar que las futuras actividades del Contador Público se desarrollarán, teniendo en cuenta la información tradicional proveniente de libros y documentos contables, como así la información que produce la dinámica con que avanza el mundo en el campo del conocimiento y la determinación del capital intelectual.

\section{ESTUDIO Y ANÁLISIS DEL CAPITAL INTELECTUAL Y DE LA CONTABI- LIDAD DEL CONOCIMIENTO}

Ya en el artículo titulado "Introducción al Universo de Paradigmas en la Contabilidad del Siglo XXI", publicado por el autor del presente artículo en la revista Quipukamayoc, Primer Semestre 2001, se recomendaba el estudio del contenido del libro CAPITAL INTELECTUAL - CONTABILIDAD DEL CONOCIMIENTO del C.P Samuel Alberto Mantilla B., publicado en Marzo de 1999, con los consiguientes comentarios y análisis de algunas áreas de más impacto; una copia del mismo está a disposición para reproducciones en el Instituto de Investigación de Ciencias Financieras y Contables de nuestra Facultad. Seguramente que a la fecha, se han publicado más libros del mismo tema y ojalá hayan llegado al Perú para incrementar nuestra biblioteca.

La Universidad de Buenos AiresArgentina, por intermedio del Instituto de Investigaciones Contables de la Facultad de
Ciencias Económicas, ha publicado en su Revista CONTABILIDAD Y AUDITORÍADiciembre 2001, el artículo titulado "Estados Contables Proyectados. Lineamientos Generales-Una Tarea Interdisciplinaria" del Dr. Mario Biondi, Director del indicado Instituto y Profesor Consultor Titular de las asignaturas de Teoría Contable y Contabilidad Patrimonial, del cual añadiremos que es un notable investigador conocido en toda América y en el mundo. En una parte del primer párrafo de la Introducción dice “... un grupo de investigadores de la Facultad de Ciencias Económicas, de la Universidad de Buenos Aires, se encuentra abocado a la tarea que incluye una serie de matices que, dificilmente, podrían ser cubiertas por una sola área del conocimiento".

También en la misma revista se publicó el artículo titulado "Valor Económico Agregado e Intangibles", de los profesores C.P Roberto Vásquez y C.P Claudia Andrea Bongianino de Salgado, con interesantes áreas de estudio. En la Introducción dice: “... Uno de los principales objetivos de las empresas es ganar dinero en el presente y a través del tiempo. Si una compañía no es capaz de generar suficiente utilidad económica estaría en duda su supervivencia. Las organizaciones que tienen bajas o nulos beneficios no son atractivas para su inversores potenciales. Tal como lo hemos desarrollado en nuestro trabajo 'Un nuevo elemento para la gestión empresarial: El Capital Intelectual', la contabilidad tradicional ha perdido su capacidad de demostrar, a través de los Estados Contables, la verdadera capacidad de las empresas para generar utilidades y proyectarse en el futuro. Una de las limitaciones más importantes es que no dispone de recursos para el reconocimiento de los activos intangibles, a pesar de que el conocimiento es uno de los factores de desarrollo más importante en la nueva economía globalizada...". 
Asimismo la Universidad Nacional de Rosario-Argentina, en la Revista de su Instituto de Investigaciones Teóricas y Aplicadas-Agosto 2001, ha publicado el artículo titulado "EL CAPITAL INTELECTUAL Y LA LLAVE DE NEGOCIO: UN ANÁLISIS COMPARATIVO". En su Introducción plantea estas dos preguntas: ¿Se trata en realidad de un nuevo elemento que aparece en las empresas?, ¿por qué se afirma que es un desafío para la Contabilidad Tradicional? En respuesta a estas interrogantes explica con muchísima claridad cuáles son los cuatro componentes del Capital Intelectual: activos de mercado, activos de propiedad intelectual, activos de infraestructura y activos centrados en el individuo; y hace mención del contenido del Estudio 7 ya mencionado en este artículo.

\section{LA IMPORTANCIA DEL CAPITAL INTELECTUAL EN LA XXIV CON- FERENCIA INTERAMERICANA DE CONTABILIDAD}

El Área 1-Investigación Contable, concentra en estos eventos interamericanos bianuales a un grupo de Contadores Públicos dedicados a la investigación de la ciencia contable. Realmente dos días de discusiones es un período corto para concentrar tantas inquietudes sobre el tema. Los asistentes nos olvidamos de horarios establecidos y las discusiones temáticas en un ambiente de plena cordialidad, son difíciles de cortar. En este último evento el Área 1-Investigación Contable, presentó el Tema 1.1-La Información Financiera frente al desafío de nuevos usuarios - con la exposición de cuatro excelentes trabajos nacionales; el Tema 1.2-Impacto de las últimas Normas Internacionales de Contabilidad en los paises de la AIC - con la exposición de cuatro trabajos nacionales, dos de ellos relacionados con la NIC 41Agricultura.

Pero, lo más interesante ha sido que por primera vez en esta Área se ha abierto el
Subtema 1.1.1. Capital Intelectual, lo que confirma la importancia que se le da al tema. Tres han sido los trabajos nacionales presentados y los asistentes se concentraron en las discusiones de los mismos, con inusitado interés.

El primer trabajo nacional discutido fue EL CAPITAL INTELECTUAL-ANÁLISIS CRÍTICO EN EL MARCO DE LAS NUEVAS NECESIDADES, presentado por tres Contadores Públicos uruguayos, que se hicieron acreedores al Segundo Premio de la Conferencia Interamericana y a merecidos elogios al contenido del trabajo expuesto de doce páginas por la profundidad de conceptos, criterios, insertaciones de acertados párrafos de diversos autores, cuadros analíticos y precisiones sobre el capital intelectual.

Los títulos y subtítulos de este interesante trabajo nacional son los siguientes:

\section{Capítulo I Introducción}

- La creación de valor en las empresas en la nueva economía.

\section{Capítulo II El debate sobre el cum- plimiento de los objeti- vos de los Estados Con- tables y su utilidad.}

\section{Capítulo III Creación y desarrollo de activos intangibles: El Capital Intelectual}

- Las fuentes de creación de valor: el Capital Intelectual.

- Definiciones de capital intelectual. Conceptualización. En la búsqueda de un concepto aceptado universalmente.

- Autores:

- L. Edvinsson y M. Malone

- OCDE (Organización para la Cooperación y el Desarrollo Económico)

- Karl Eric Sveiby 
- Baruch Lev

- Toni Stewart

- Annie Brooking

- Eduardo Bueno Campos

- Principales activos intangibles definidos por la corriente de capital intelectual

- Modelos de medición del Capital Intelectual

- Navegador de Skandia

- Enfoque financiero (20 índices)

- Enfoque de proceso (19 índices)

- Enfoque humano (22 índices)

- Enfoque cliente (20 índices)

- Enfoque de renovación y desarrollo (32 índices)

1. Lo que cambia:

- Un nuevo informe de Capital Intelectual

- Incrementos de valor: Inversiones en Capital Intelectual

2. Lo que sigue igual:

- No cambios; en las técnicas ni en los principios contables

- Modelos de medición de Capital Intelectual de Karl Sueiby: el monitor de activos intangibles

- Propuestas tradicionales para medir la creación de valor

\section{Capítulo IV La visión de la profesión}

1. ¿Los estados contables o sus notas revelan todos los hechos pasados que puedan indicar la existencia de una fuente generadora de valor?

2. ¿Los estados contables consideran la influencia de las actividades desarrolladas dentro de las empresas como fuente generadora de valor?

- Identificabilidad

- Control

- Beneficios económicos futuros
3. ¿La profesión contable permite la valorización de aquellos activos intangibles de reconocimiento admitido siguiendo criterios de medición alineados con la cuantificación de su potencialidad como fuente de generación de valor?

4. ¿Permiten los estados contables extraer conclusiones válidas sobre la evolución económica y financiera de las empresas basadas en una razonable correlación entre los ingresos y gastos asociados a la utilización del Capital Intelectual?

\section{Capítulo V. Nuestra propuesta:Modelo de Información Comple- mentaria sobre Capital Intelectual}

\section{Capítulo VI Guía de discusión}

\section{Capítulo VII Conclusiones}

1. Necesidad de incorporar información sobre el Capital Intelectual

2. Sobre el futuro de la profesión

3. Cambios a las normas contables

\section{Bibliografía}

\section{CAPITAL INTELECTUAL-INFORMACIÓN COMPLEMENTARIA A LOS ESTADOS CONTABLES}

El Capítulo V del mencionado trabajo nacional interamericano, presenta el cuadro titulado CAPITAL INTELECTUALINFORMACIÓN COMPLEMENTARIA A LOS ESTADOS CONTABLES, que se presenta en la página 34 de este artículo como una excelente aportación al desarroIlo de la información contable; merece los siguientes comentarios sobre su estructura y contenido:

1. Es la primera vez que los Contadores Públicos tenemos la posibilidad de 
brindar a una empresa una información cuantitativa y cualitativa complementaria sobre Capital Intelectual, debidamente interconectada con indicadores con base contable $y$ extracontable. Esta interconexión es un logro inobjetable que otorga un gran mérito al cuadro diseñado.

2. Los componentes del Capital Intelectual están expuestos en tres categorías: Mercado; Innovación y Tecnología; y Competencias del personal.

En cada categoría se observan columnas de: unidad de medida, base contable, base extracontable y base mixta. Estas tres últimas columnas comparativas de un año a otro, le dan al cuadro un valor agregado para efectuar análisis y evaluaciones de carácter financiero y económico.

3. Estamos, por lo tanto, frente a una nueva era de información contable y extracontable, que nace en el continente americano. Debemos considerar esta información complementaria como la primera puerta que se abre en el conglomerado de conceptos, criterios y análisis teóricos que están rodeando a los temas de Capital Intelectual y Contabilidad del Conocimiento.

4. Por último tenemos un reto los Contadores Públicos, en plantear los mejores procedimientos operativos para anotar valores contables y extracontables en la información complementaria sobre Capital Intelectual, concentrados con auténtica veracidad y confiabilidad.

\section{CONTENIDOS DE TRABAJOS NACIONALES INTERAMERICANOS SOBRE CAPITAL INTELECTUAL Y CONTABILIDAD DEL CONOCIMIENTO}

En la conferencia interamericana última (noviembre 2001), se expusieron también los siguientes trabajos nacionales sobre el tema:
- Los Intangibles y la Información Contable

Que analiza a fondo la problemática de los intangibles y plantea también un Proyecto de Información Complementaria sobre Intangibles, afirmando que “... El Capital Intelectual representaría entonces la suma de todos aquellos intangibles presentes en la empresa que se identifican con el valor creado por la investigación y desarrollo, la innovación, las prácticas organizacionales internas, las relaciones y vínculos con los agentes del contexto y la acción de los recursos humanos que, tradicionalmente, no son reconocidos en su totalidad por la contabilidad financiera...".

- Insuficiencia de la Teoría Contable Normativa ante la Demanda de Información de los Mercados Financieros. Los Recursos Humanos y el capital Intelectual

Que presenta una propuesta de evaluación del capital Intelectual que podría integrar el Patrimonio de un Ente y que merece un estudio y análisis completo para conocer la posición del autor de este trabajo nacional, quien es el Dr. Mario Biondi ya presentado en este artículo.

\section{EL XVI CONGRESO MUNDIAL DE CONTABLES EN HONG KONG- NOVIEMBRE 2002: EL CONTABLE Y LA ECONOMÍA BASADA EN EL CONOCIMIENTO}

Del 18 al 21 de noviembre del 2002 se realizará en la ciudad de Hong Kong, el XVI Congreso Mundial de Contables, cuyo Programa Técnico precisa que se llevará a cabo Tres Sesiones Plenarias y Trece Grupos de Trabajo, que estarán centrados en el tema del Congreso: "El Contable y la economía basada en el Conocimiento". 


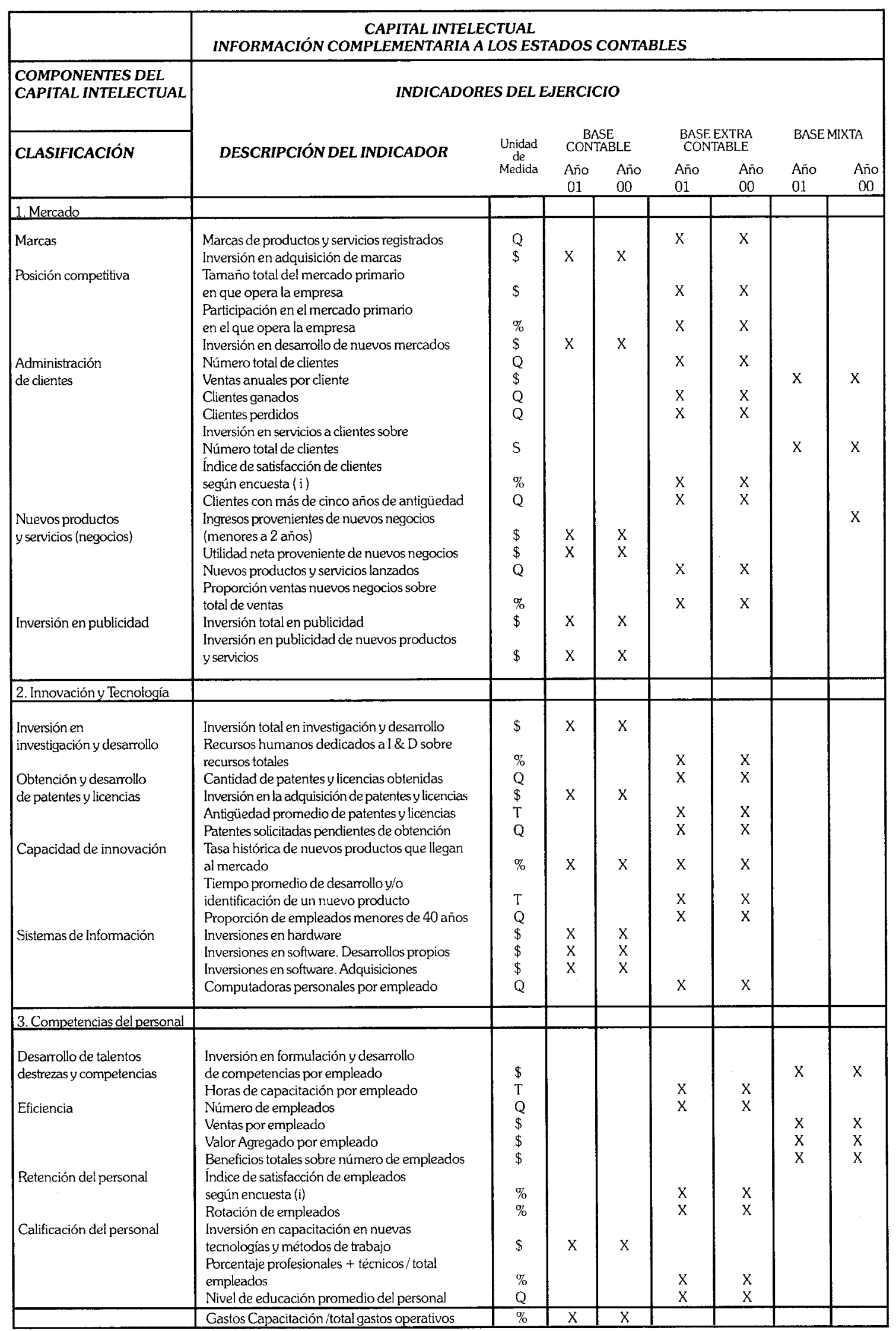

Referencia sobre la unidad de medida: T Tiempo, \% Porcentaje, $Q$ Cantidad, \$ Importe (i), Incorporar ficha técnica de las encuestas realizadas. 
Las tres Sesiones Plenarias plantearán los siguientes interrogantes:

- Sesión Plenaria 1

- ¿Qué oportunidad ofrece la economía basada en el conocimiento?

- ¿Cómo debemos redefinir "el contable" y "la contabilidad" para el Siglo XXI?

- Sesión Plenaria 2

Sobrevivir y profesar en una economía basada en el conocimiento:

- ¿De qué manera las nuevas iniciativas de IFAC ayudan al posicionamiento de la profesión de contabilidad para triunfar en la economía basada en el conocimiento?

- Sesión Plenaria 3

Sobrevivir y prosperar en una economía basada en el conocimiento:

- ¿Qué cuestiones prácticas hay que superar antes que los contables puedan alcanzar su nueva identidad?

Para quienes desean mayor información, pueden visitar la página web del indicado Congreso en www.wco@2002.com; o dirigirse a la Federación de Colegios de Contadores Públicos del Perú.

Analizando los interrogantes ya planteados en las tres Sesiones Plenarias, observamos que son sumamente inquietantes; por cuanto tenemos escasa bibliografía sobre la contabilidad del conocimiento y poco interés en el estudio de este apasionado tema, tanto a nivel profesional como en los estudios universitarios de contabilidad del Perú.

Entonces ¿qué debemos hacer los Contadores Públicos y los estudiantes universitarios en formación como futuros Contadores Públicos, frente al reto planteado? En primer lugar estudiar la teoría del Capital Intelectual y de la Contabilidad del
Conocimiento; luego conocer los avances internacionales que se han logrado $y$ proponen aplicaciones en el campo contable del Perú.

\section{CONCLUSIONES}

1. Es necesario fijar una primera obligación para los Contadores Públicos del Perú. Esta obligación es el estudio y comprensión a cabalidad de todo lo escrito y comentado sobre $\mathrm{Ca}$ pital Intelectual y Contabilidad del Conocimiento.

Luego, se requiere plantear el compromiso de difusión profesional a nivel de colegios profesionales $y$ de facultades que comprometidas en la formación de futuros Contadores Públicos, para servir con eficiencia a la sociedad peruana.

2. Es también un deber para los Contadores Públicos y estudiantes universitarios de todas las facultades del Sistema de la Universidad Peruana, exigir el dictado de cursos, conferencias y seminarios sobre Capital Intelectual y Contabilidad del Conocimiento, tanto a los Colegios Profesionales como a sus propias universidades, para asimilar las innovaciones contables y extracontables que se están presentando. Así, los Contadores Públicos del Perú estarán capacitados en ofrecer nuevos servicios profesionales competitivos, aportando planteamientos de información contable de avanzada. De igual forma, los estudiantes universitarios, futuros Contadores Públicos al salir de la universidad, tendrán una adecuada preparación sobre estos interesantes temas profesionales y estarán preparados para enfrentarse al reto de información futura que exigirán las empresas que hayan captado la importancia de recibir información contable basada en estos temas. 
3. También tiene prioridad conocer los avances logrados sobre el Capital Intelectual y la Contabilidad del Conocimiento, realizando serios estudios de los trabajos nacionales que tratan estos temas; y de los artículos y libros que difundan los alcances logrados en las aplicaciones contables y extracontables a nivel empresarial.
4. El reto más ambicioso será buscar la mejor aplicación del Capital Intelectual y la Contabilidad del Conocimiento en la economía peruana, para producir mayor información confiable, que permita el progreso y crecimiento financiero y económico de las empresas del Perú, el incremento de puestos de trabajo y la mejora de los niveles económicos de la población peruana. 